\title{
Subtypes of nurses' mental workload and interaction patterns with fatigue and work engagement during coronavirus disease 2019 (COVID-19) outbreak: A latent class analysis
}

\section{JING WU}

Shanghai University of Traditional Chinese Medicine

\section{HUSHENG LI}

Shanghai University of Traditional Chinese Medicine https://orcid.org/0000-0003-1722-0838

\section{ZHAOHUI GENG}

Shanghai University of Traditional Chinese Medicine

\section{YANMEI WANG}

Shanghai Municipal Hospital of TCM affiliated to Shanghai University of TCM

\section{XIAN WANG}

Shanghai Municipal Hospital of TCM affiliated to Shanghai University of TCM

JIE ZHANG ( $\sim$ allydata@126.com )

University of TCM

\section{Research article}

Keywords: nurses, mental workload, latent class analysis, fatigue, work engagement, COVID-19

Posted Date: September 21st, 2020

DOI: https://doi.org/10.21203/rs.3.rs-70155/v1

License: (c) (i) This work is licensed under a Creative Commons Attribution 4.0 International License.

Read Full License

Version of Record: A version of this preprint was published at BMC Nursing on October 22nd, 2021. See the published version at https://doi.org/10.1186/s12912-021-00726-9. 


\section{Abstract \\ Background}

Nurses play critical roles when providing health care in high-risk situations, such as during the COVID-19 outbreak. However, no previous study had systematically assessed nurses' mental workloads and its interaction patterns with fatigue, work engagement and COVID-19 exposure risk.

\section{Methods}

A cross-sectional study was conducted via online questionnaire. The NASA Task Load Index, Fatigue Scale-14, and Utrecht Work Engagement Scale were used to assess nurses' mental workload, fatigue and work engagement, respectively. A total of 1337 valid questionnaires were received and analyzed. Nurses were categorized into different subgroups of mental workload via latent class analysis (LCA). Crosssectional comparisons, analysis of covariance (ANCOVA), and multivariate (or logistic) regression were subsequently performed to examine how demographic variables, fatigue and work engagement differ among nurses belonging to different subgroups.

\section{Results}

Three latent classes were identified based on the responses to mental workload assessment: Class1 low workload perception \& high self-evaluation group $(n=41,3.1 \%)$; Class 2 - medium workload perception \& medium self-evaluation group $(n=455,34.4 \%)$; and Class 3 - high workload perception \& low self-evaluation group $(n=841,62.5 \%)$. Nurses belonging into class 3 were most likely to be older and have longer professional years, and displayed higher scores of fatigue and work engagement compared with the other latent classes $(p<0.05)$. Multivariate analysis showed that high cognitive workload increased subjective fatigue, and mental workload may be positively associated with work engagement. Group comparison results indicated that COVID-19 exposure contributed to significantly higher mental workload levels.

\section{Conclusions}

The complex scenario for the care of patients with infectious diseases, especially during an epidemic, raises the need for improved consideration of nurses' perceived workload, as well as their physical fatigue, work engagement and personal safety when working in public health emergencies.

\section{Background}

The coronavirus diseases 2019 (COVID-19) outbreak, with its rapid spread and high mortality rate, has caused a significant burden to the health care system. The infection rate of COVID-19 is significantly 
higher among health care workers than any other group (Houghton et al., 2020). Although newly confirmed cases of coronavirus in China have declined significantly since its peak, medical professionals still face considerable physical and psychological stress.

Nurses are at the forefront in providing health care for serious or critical COVID-19 patients that require hospitalization. It has been reported that nearly $17 \%$ of COVID-19 patients received invasive mechanical ventilation therapy and up to $2 \%$ of required the use of ECMO (Zhou et al., 2020). As a result, a large proportion of nurses have been faced with complex and demanding tasks that require significant mental concentration and effort during day-to-day work. Mental workload, also known as cognitive workload, is defined as the amount of mental effort and operator's ability involved in completing a task (Collet et al., 2014). The study of mental workload is one of the most important variables in psychology and behavior for understanding job performance. Studies have shown that a higher mental workload results in a decreased capacity for attention, and increases the risk of slow responses, ultimately contributing to workplace errors (Jaquess et al., 2018). The mental workload level, and its association with a particular task, has been studied in a number of healthcare disciplines, including anesthesiology, emergency medicine, surgery, and nursing. However, there have been significantly fewer studies on nurses' mental workload under special working conditions, for example, nurses working in ICU and emergency departments, especially during a health crisis (Tubbs-Cooley et al., 2018; Tubbs-Cooley et al., 2019). To our knowledge, no previous study has systematically assessed the mental workload of nurses and its relevant factors during a large-scale infectious public health crisis such as the COVID-19 outbreak.

The most widely used instrument for assessment of overall subjective workload is the National Aeronautics and Space Administration Task Load Index (NASA-TLX), which contains a total of six dimensions: mental, physical, and temporal task demands, as well as effort, frustration, and perceived performance (Charles et al., 2019). The NASA-TLX has been validated and widely used as an assessment tool in a healthcare setting. Recent studies showed that factors such as an increased workload, interruptions, level of personal experience, and stress have the ability to influence mental workload, which can subsequently have an impact on nurses' critical decision making and patients' safety (Happell et al., 2013). However, ergonomics studies have suggested that the measurement of mental workload using the NASA-TLX requires taking into account each of its individual dimensions, rather than simply assessing of the global score (Galy et al., 2018).

The aim of this study was to clarify the subgroups of nurses' mental workload via latent class analysis. We also explored the association between the different subgroups based on various demographic or psychological factors. We hypothesized that nurses can be separated into different subgroups based on the assessment of NASA-TLX, and that key factors including demographic characteristics, fatigue, and work engagement differed across different subgroups. Specifically, we examined the risk of COVID-19 exposure in order to determine the discrepancy in self-perception and perceived workload during this global health crisis. This study is the first of its type that has been conducted in China, and provides an important reference point for comparison with future international studies. 


\section{Methods}

\section{Design}

This study employed a cross-sectional design using data from an online questionnaire survey.

\section{Data sources and sampling}

Full-time nurses who worked during the COVID-19 outbreak were invited to complete an anonymous and self-rated questionnaire in March 2020. Nursing students and long-term off-duty nurses were excluded, as they were not at work during the outbreak and cannot accurately reflect the mental workload faced.

All study participants were recruited from six general hospital hospitals in China that each contained over 800 beds per hospital. Respondents included those who have been working in intensive care unit, fever clinic, emergency department, outpatient department, general wards or nursing departments, as well as those who were sent to Wuhan by the hospital for assistance during the outbreak.

Questionnaires were distributed to 1551 nurses via online survey tools in order to facilitate the study across different hospitals and regions and avoid unnecessary face-to-face contact during the outbreak. Researchers who took part in the data collection process directly or indirectly had no conflicting interests so as to maximize voluntary participation and protect confidentiality. Questionnaires with more than $10 \%$ of items left unanswered or containing logical errors were excluded from the study. Finally, a total of 1337 questionnaires were available for further analysis.

\section{Measures}

In order to analyze the mental workload of nurses by socio-demographic characteristics, the baseline characteristics were recorded including gender, age, marital status, parental status, educational level, professional title, and years of nursing experience. Furthermore, COVID-19 work-related information were recorded including degree of exposure to the virus, actual working hours per day, work experience in public health emergencies, and willingness to care for or assist patients during the COVID-19 outbreak.

We assessed the participants' mental workload using the NASA Task Load Index (NASA-TLX) (Hart et al., 1988; Hart, 2006). The current version is made up of six items and reflects the mental workload based on two underlying dimensions: workload perception and self-evaluation. Response items including mental, physical and temporal task demands, effort, and frustration, were marked on a scale ranging from 0 (very low) to 20 (very high), as well as performance, which ranged from 0 (perfect) to 20 (failure). By taking a non-weighted (raw) summed numeric score of the items, the overall mental workload was calculated from a minimum of 0 , up to a maximum of 120 . Higher scores indicated nurses who faced heavier mental workloads during the COVID-19 outbreak. A previous study demonstrated the scale's validity and reliability in a Chinese population, with a Cronbach's alpha score of 0.782 (Liang, 2019).

The Fatigue Scale-14 (FS-14) (Chalder et al., 1993) was designed to reflect the recent fatigue symptoms and severity of participants. The dichotomized survey is comprised of fourteen items ranging from 
measurements of physical fatigue (eight items) and mental distress (six items). Higher scores for a given scale indicated participants who had stronger fatigue. A previous study demonstrated the scale's validity and reliability in a Chinese population, with a Cronbach's alpha score of 0.77 (Xu et al., 2006).

Work engagement can be explained by a positive and fulfilling state of mind in relation to the work being performed. In the study, we collected relevant data via the Utrecht Work Engagement Scale (UWES) (Fang et al., 2008), which is a self-reporting inventory composed of seventeen items based on three components: vigor (six items), dedication (five items), and absorption (six items). Each item was measured on a seven-point Likert scale ranging from 0 (never) to 6 (always). Higher scores indicated nurses who had greater work engagement. The results of a Spanish study showed that the internal consistency coefficients of vitality, dedication and concentration scale were $0.79,0.89$ and 0.72 , respectively (Schaufeli et al., 2002).

\section{Statistical analyses}

We performed latent class analysis (LCA) using Mplus version 7.2, where we explored homogenous subgroups in a heterogeneous group and then observed categorical variables in each subgroup (Chen et al., 2015). To determine the most appropriate latent class model, the combination of model fitting indexes was considered, including Akaike information criterion (AIC), Bayesian information criterion (BIC), samplesize adjustment BIC (ABIC), the Lo-Mendell-Rubin likelihood ratio test (LMR-LRT), and entropy, along with the research interpretability. The best-fit model selected was based on the following criteria: (1) LMRLRT $\leq 0.05$; (2) higher entropy value, and lower information criterion score; (3) interpretability.

After examining the best-fit model of latent classes based on nurses' conditional response of the NASATLX, the data was further adjusted by demographic characteristics, compared the score of work engagement and fatigue through covariance analysis (ANCOVA) and multivariate (or logistic) regression across the subgroups. Simultaneously,we used a multiple correspondence analysis (MCA) model to explore the potential relationship between latent classes and various influence factors based on a visualized factor map. Statistical significance of all tests was set as two-sided $P$ value $<0.05$. All analyses of data were conducted using SPSS statistical version 26.0.

\section{Results}

\section{Latent classes of nurses' mental workload response}

In order to classify and identify the optimal model, we extracted and compared the model solutions from the two-class to six-class models. The model diagnostics for the overall samples were summarized in Table 1. The five-class and six-class models of LMR-LRT were not significant, and thus neither was considered. Whereas the two-class model had the highest information criterion score and the four-class model had the lowest entropy value, both models had shortcomings. We thus identified that the threeclass model for nurses' mental workload conditional response provided the best overall model fit, 
including a statistically significant LMR-LRT p-value, the best entropy, and relatively lower information criterion scores.

Table 1

Latent class model fit index

\begin{tabular}{|llllllll|}
\hline Model & AIC & BIC & ABIC & $\begin{array}{l}\text { LMR LR } \\
\text { test P- } \\
\text { value }\end{array}$ & $\begin{array}{l}\text { ALMR LR } \\
\text { test P-value }\end{array}$ & $\begin{array}{l}\text { BLRT } \\
\text { P-value }\end{array}$ & Entropy \\
\hline $\begin{array}{l}\text { 2-class } \\
\text { LCA }\end{array}$ & 44084.315 & 44183.081 & 44122.726 & 0.0000 & 0.0000 & 0.0000 & 0.839 \\
$\begin{array}{l}\text { 3-class } \\
\text { LCA }\end{array}$ & 43617.022 & 43752.175 & 43669.584 & 0.0412 & 0.0434 & 0.0000 & 0.873 \\
\hline $\begin{array}{l}\text { 4-class } \\
\text { LCA }\end{array}$ & 43308.326 & 43479.866 & 43375.040 & 0.0060 & 0.0065 & 0.0000 & 0.808 \\
\hline $\begin{array}{l}\text { 5-class } \\
\text { LCA }\end{array}$ & 43175.542 & 43383.470 & 43256.407 & 0.1582 & 0.1632 & 0.0000 & 0.829 \\
\hline $\begin{array}{l}\text { 6-class } \\
\text { LCA }\end{array}$ & 43029.236 & 43273.551 & 43124.252 & 0.4491 & 0.4562 & 0.0000 & 0.840 \\
\hline
\end{tabular}

The profiles of the three latent classes based on the LCA results were listed in Table 2, while a line diagram representation was shown in Fig. 1. The x-axis represented six items of NASA-TLX and the y-axis showed the probability of conditional response. Higher levels of mental demand, physical demand, temporal demand, and effort indicated higher perceptive workload, while higher scores of performance and frustration indicated lower self-evaluation scores. Nurses in class 1 had the lowest workload perception and relatively high self-evaluation, with a total of $3.1 \%(n=41)$ of all participants belonging to this group. We therefore classified nurses belonging to class 1 as the "low workload perception \& high self-evaluation group". Nurses in class 2 tended to have average scores of workload perception and selfevaluation, but had no anomalies in each of their mental workload scores. We therefore categorized nurses belonging to class 2 as the "medium workload perception \& medium self-evaluation group", with a total of $34.4 \%(n=455)$ of all participants belonging to this group. Nurses in class 3 displayed high scores of workload perception, but relatively lower scores of self-evaluation. Thus, nurses belonging to class 3 were classified as "high workload perception \& low self-evaluation group", which comprised a total of $62.5 \%(n=841)$ of all participants. 
Table 2

The profiles of the classification

\begin{tabular}{|lllllll|}
\hline $\begin{array}{l}\text { Model Mean } \\
\text { Model }\end{array}$ & $\begin{array}{l}\text { Mental } \\
\text { Demand }\end{array}$ & $\begin{array}{l}\text { Physical } \\
\text { Demand }\end{array}$ & $\begin{array}{l}\text { Temporal } \\
\text { Demand }\end{array}$ & Performance & Effort, & Frustration \\
\hline $\begin{array}{l}\text { Class } 1(\mathrm{~N}= \\
41,3.1 \%)\end{array}$ & 4.622 & 4.283 & 5.625 & 6.148 & 11.083 & 4.708 \\
\hline $\begin{array}{l}\text { Class } 2(\mathrm{~N}= \\
455,34.0 \%)\end{array}$ & 11.515 & 13.659 & 12.091 & 6.874 & 13.810 & 8.922 \\
\hline $\begin{array}{l}\text { Class } 3(\mathrm{~N}= \\
841,62.9 \%)\end{array}$ & 16.680 & 18.675 & 17.407 & 3.346 & 18.125 & 11.057 \\
\hline
\end{tabular}

${ }^{a}$ Class 1: low workload perception \& high self-evaluation group; Class 2: medium workload perception \& medium self-evaluation group; Class 3: high workload perception \& low self-evaluation group

\section{Differences in the latent classes by characteristics}

The statistical description for distributions of each demographic group within latent classes were listed in Table 3. A total of 1337 subjects were enrolled in the study, including 1278 females (95.59\%) and 59 males. There were few differences based on gender. Univariate analysis results among the groups showed that participants belonging to class 1 were mostly aged $<28(56.10 \%)$ and have nursing profession $\leq 5$ year $(46.34 \%)$. Class 2 and 3 both had a high proportion of bachelor's degree or above ( $57.36 \%$ and $63.85 \%$, respectively), with class 2 being younger overall compared to class 3 . Participants belonging to class 3 were most aged $28 \sim 38$ (41.14\%), married (71.22\%), have children (63.26\%), have nursing profession $>10$ years $(47.21 \%)$, and have high COVID-19 exposure $(p<0.05$ for all). 
Table 3

Demographic characteristics of mental workload subgroups

\begin{tabular}{|c|c|c|c|c|c|}
\hline & $\begin{array}{l}\text { Overall } n= \\
1337\end{array}$ & $\begin{array}{l}\text { Class } 1 \\
\mathrm{n}=\mathbf{4 1}\end{array}$ & $\begin{array}{l}\text { Class } 2 \\
\mathrm{n}=455\end{array}$ & $\begin{array}{l}\text { Class } 3 \\
\mathrm{n}=841\end{array}$ & $p$ \\
\hline Gender (female) & 1278 (95.59) & $\begin{array}{l}40 \\
(97.56)\end{array}$ & $\begin{array}{l}428 \\
(94.07)\end{array}$ & $810(96.31)$ & 0.140 \\
\hline Age (Yrs) & & & & & 0.000 \\
\hline$₫ 28$ & $496(37.10)$ & $\begin{array}{l}23 \\
(56.10)\end{array}$ & $\begin{array}{l}196 \\
(43.08)\end{array}$ & $\begin{array}{l}277 \\
(32.94)\end{array}$ & \\
\hline $28-38$ & $523(39.12)$ & $9(21.95)$ & $\begin{array}{l}168 \\
(36.92)\end{array}$ & $\begin{array}{l}346 \\
(41.14)\end{array}$ & \\
\hline$₫ 38$ & $318(23.78)$ & $9(21.95)$ & $91(20.00)$ & $\begin{array}{l}218 \\
(25.92)\end{array}$ & \\
\hline Education & & & & & 0.011 \\
\hline bachelor's degree & $817(61.11)$ & $\begin{array}{l}19 \\
(46.34)\end{array}$ & $\begin{array}{l}261 \\
(57.36)\end{array}$ & $\begin{array}{l}537 \\
(63.85)\end{array}$ & \\
\hline Non-bachelor' degree & $520(38.89)$ & $\begin{array}{l}22 \\
(53.66)\end{array}$ & $\begin{array}{l}194 \\
(42.64)\end{array}$ & $\begin{array}{l}304 \\
(36.15)\end{array}$ & \\
\hline Nursing profession year & & & & & 0.000 \\
\hline 85 & 419 (31.14) & $\begin{array}{l}19 \\
(46.34)\end{array}$ & $\begin{array}{l}169 \\
(37.14)\end{array}$ & $\begin{array}{l}231 \\
(27.47)\end{array}$ & \\
\hline $5-10$ & $355(26.55)$ & $8(19.51)$ & $\begin{array}{l}134 \\
(29.45)\end{array}$ & $\begin{array}{l}213 \\
(25.33)\end{array}$ & \\
\hline$\$ 10$ & $563(42.11)$ & $\begin{array}{l}14 \\
(34.15)\end{array}$ & $\begin{array}{l}152 \\
(33.41)\end{array}$ & $\begin{array}{l}397 \\
(47.21)\end{array}$ & \\
\hline Marital status(married) & $899(67.24)$ & $\begin{array}{l}22 \\
(53.66)\end{array}$ & $\begin{array}{l}278 \\
(61.10)\end{array}$ & $\begin{array}{l}599 \\
(71.22)\end{array}$ & 0.000 \\
\hline Child of all ages (Yes) & $784(58.64)$ & $\begin{array}{l}19 \\
(46.34)\end{array}$ & $\begin{array}{l}233 \\
(51.21)\end{array}$ & $\begin{array}{l}532 \\
(63.26)\end{array}$ & 0.000 \\
\hline \multicolumn{6}{|c|}{$\begin{array}{l}\text { Degree of Exposure to COVID- } \\
19\end{array}$} \\
\hline Low COV Exp & $820(61.33)$ & $\begin{array}{l}28 \\
(68.29)\end{array}$ & $\begin{array}{l}276 \\
(60.66)\end{array}$ & $\begin{array}{l}516 \\
(61.36)\end{array}$ & 0.016 \\
\hline
\end{tabular}

${ }^{a}$ Class 1: low workload perception \& high self-evaluation group; Class 2: medium workload perception \& medium self-evaluation group; Class 3: high workload perception \& low self-evaluation group

bLow COV Exp, Low COVID-19 exposure; Med COV Exp, Medium COVID-19 exposure; High COV Exp, High COVID-19 exposure 


\begin{tabular}{|c|c|c|c|c|c|}
\hline & $\begin{array}{l}\text { Overall } n= \\
1337\end{array}$ & $\begin{array}{l}\text { Class } 1 \\
\mathrm{n}=\mathbf{4 1}\end{array}$ & $\begin{array}{l}\text { Class } 2 \\
n=455\end{array}$ & $\begin{array}{l}\text { Class } 3 \\
\mathrm{n}=841\end{array}$ & $p$ \\
\hline Med COV Exp & 389 (29.09) & $9(21.95)$ & $\begin{array}{l}145 \\
(31.87)\end{array}$ & $\begin{array}{l}241 \\
(27.94)\end{array}$ & \\
\hline High COV Exp & $128(9.57)$ & $4(9.76)$ & $34(7.47)$ & $90(10.07)$ & \\
\hline \multicolumn{6}{|c|}{$\begin{array}{l}\text { aClass 1: low workload perception } \& \text { high self-evaluation group; Class } 2 \text { : medium workload perception } \\
\& \text { medium self-evaluation group; Class } 3 \text { : high workload perception \& low self-evaluation group }\end{array}$} \\
\hline \multicolumn{6}{|c|}{$\begin{array}{l}\text { bLow COV Exp, Low COVID-19 exposure; Med COV Exp, Medium COVID-19 exposure; High COV Exp, } \\
\text { High COVID-19 exposure }\end{array}$} \\
\hline
\end{tabular}

The mean scores of FS-14 among subjects were $6.94 \pm 2.43$, and UWES were $57.75 \pm 11.47$, as shown in Table 4. There were significant differences among the three interaction classes, including the scores of FS-14, UWES, and their dimension scores ( $p<0.05$ for all), except for the measurement of mental distress. The results of the multivariate regression analyses that predicted the outcome variables of UWES and FS14 by the three latent classes were shown in Table 5. We found significant differences related to fatigue and work engagement among the different classes of mental workload. In particular, nurses in class 3 were significantly more likely to report higher scores of work engagement relative to those in class 2 , indicating that nurses who had self-perceived high workload and low self-evaluation were likely to feel more engaged at work compared to nurses who had average workload perception and self-evaluation. Similar patterns were found in the outcomes of FS-14, specifically, nurses in class 3 reported a significantly higher level of fatigue than those in class 2 . In other words, the nurses' workload perception during the COVID-19 outbreak may be positively associated with their fatigue status. 
Table 4

The mean scores of FS-14 and UWES of mental workload subgroups

\begin{tabular}{|llllll|}
\hline & Overall $\mathbf{n = 1 3 3 7}$ & Class $\mathbf{1}$ & Class $\mathbf{2}$ & Class $\mathbf{3}$ & $\boldsymbol{p}$ \\
\hline FS-14 & & $\mathbf{n = 4 1}$ & $\mathbf{n = 4 5 5}$ & $\mathbf{n = 8 4 1}$ & \\
\hline physical fatigue & $4.89 \pm 1.43$ & $4.61 \pm 1.53$ & $4.65 \pm 1.55$ & $5.04 \pm 1.34$ & 0.028 \\
\hline mental fatigue & $2.05 \pm 1.18$ & $2.07 \pm 1.06$ & $1.99 \pm 1.14$ & $2.08 \pm 1.09$ & 0.075 \\
\hline UWES & $57.75 \pm 11.47$ & $61.12 \pm 12.82$ & $54.43 \pm 9.70$ & $59.37 \pm 12.02$ & 0.000 \\
\hline vigor & $19.59 \pm 4.72$ & $20.73 \pm 4.02$ & $18.60 \pm 3.68$ & $20.07 \pm 3.93$ & 0.000 \\
\hline dedication & $19.68 \pm 4.71$ & $21.07 \pm 5.19$ & $18.54 \pm 4.22$ & $20.22 \pm 3.85$ & 0.000 \\
\hline absorption & $18.47 \pm 3.96$ & $19.31 \pm 4.35$ & $17.29 \pm 3.29$ & $19.07 \pm 4.16$ & 0.000 \\
\hline $\begin{array}{l}\text { aClass 1: low workload perception \& high self-evaluation group; Class 2: medium workload perception } \\
\text { \& medium self-evaluation group; Class 3: high workload perception \& low self-evaluation group }\end{array}$ \\
\hline
\end{tabular}

Table 5

Results of multivariate regressions predicting fatigue and work engagement by mental workload subgroups

\begin{tabular}{|c|c|c|c|c|}
\hline & \multicolumn{2}{|l|}{ UWES } & \multicolumn{2}{|l|}{ FS-14 } \\
\hline & $\mathrm{B}(\mathrm{SE})$ & $p$ & $\mathrm{~B}(\mathrm{SE})$ & $p$ \\
\hline Class 3 vs. 1 & $-1.085(1.283)$ & 0.198 & $0.200(0.271)$ & 0.541 \\
\hline Class 3 vs. 2 & $4.750(0.892)$ & $0.000^{\star *}$ & $0.428(0.200)$ & $0.033^{*}$ \\
\hline Class 2 vs. 1 & $-6.761(2.285)$ & $0.003^{* *}$ & $-0.047(0.575)$ & 0.735 \\
\hline \multicolumn{5}{|c|}{$\begin{array}{l}\text { a Class 1: low workload perception \& high self-evaluation group; Class 2: medium workload } \\
\text { perception \& medium self-evaluation group; Class 3: high workload perception \& low self-evaluation } \\
\text { group; B, unstandardized coefficient; SE, standard error. }\end{array}$} \\
\hline \multicolumn{5}{|c|}{$\begin{array}{l}\text { bAdjusted by gender, years in nursing profession, having a bachelor's degree, marital status, and } \\
\text { having children. }\end{array}$} \\
\hline${ }^{\star} p<0.05 .{ }^{*} p$ & & & & \\
\hline
\end{tabular}

We further entered significant categorical variables into the multiple correspondence analysis (MCA) model to evaluate the potential association among various latent classes. The continuous variables were converted into categorical variables according to the different quartiles (e.g., age, nursing profession year, FS-14, and UWES). In the MCA plot, the distance between any row or column points reflected the similarity (or non-similarity) between the variables. Categorical variables with similar profiles were visualized on a 
coordinate chart (Fig. 2), which showed that UWES > 67, FS-14 > 7, high \& medium COVID-19 exposure, and having children, were associated with class 3; UWES $=48 \sim 67$, FS-14 $<7$, and low COVID-19 exposure, were correlated with class 2; UWES $<48$ and not having a bachelor's degree, were associated with class 1 , respectively.

\section{Discussion}

In this study, we identified three separate mental workload subtypes among nurses who had worked in hospitals during the COVID-19 outbreak. Of the 1337 nurses, the majority (62.5\%) belonged to class 3 "high workload perception \& low self-evaluation group", who are most likely to be associated with longer professional years, more fatigue, better work engagement and higher COVID-19 exposure.

Self-perceived stress and mental demands are often underestimated using conventional objective workload indices, such as patient-to-nurse staffing ratios or patient acuity scores. The NASA-TLX is frequently used in emergency and Critical Care Units as a subjective measure of hospital staff workload and is supposed to be a stronger predictor of patient outcomes (Legenza et al., 2019). The generic version of the NASA-TLX contains six items that are marked on a twenty-point scale (Felton et al., 2012). Studies have suggested that a 4-item NASA-TLX may be more suitable for measuring nurse workload, however there is still controversy regarding the application of 4-item or 6-item versions of NASA-TLX in the clinical setting (Galy et al., 2018; Charles et al., 2019). Our current study utilized the 6-item version of NASA-TLX in order to assess different aspects of nurse workload. Our results demonstrated higher levels of perceived workload than previous studies in terms of the overall score of NASA-TLX (Young et al., 2008; Hoonakker et al., 2011), especially evident in those in class 3, which can be attributed to the heavier overload faced by Chinese nurses during the COVID-19 outbreak. Another characteristic of nurses in class 3 was the low self-evaluation scores, meaning that they felt more frustrated while working in the hospitals, and this can be partly explained by tense patient-nurse relationships and the higher risk of COVID-19 exposure during the outbreak. During working hours, nurses were required to wear personal protective equipment (PPE) for 6 hours or more of work, and coupled with the frequent and timeconsuming process of putting on and taking off PPE, would have had adverse effects on the nurses' mental burden (Huang et al., 2020).

Comparisons of demographic variables across different subgroups showed that nurses who were older and had longer professional years had a higher probability of belonging to class 3 . This result is consistent with a previous study that showed employees over 36 years of age had heavier work pressure than those younger than 36 (Donders et al., 2012). How someone experiences workload may directly influence their own actions and responses. Older nurses are often imposed with increased responsibility, which may in turn lead to a heavier workload. However, in contrast to other previous findings (TubbsCooley et al., 2018), our study showed that there were no gender differences between different subgroups, which may be attributed to the substantially lower proportions of male nurses. 
Mental demand is often significantly higher when performing more difficult tasks, and therefore maximizing the available mental resources during difficult situations is crucial, especially when quick decisions need to be made. In order to further assess the impact of the COVID-19 outbreak exposure on nurses' mental workload, we stratified all subjects to three categories according to the risk of virus exposure across different nursing units. Nurses with the highest exposure risk were those who had direct contact with confirmed COVID-19 positive patients (Huang et al., 2020). Group comparison results indicated that COVID-19 exposure contributed to significantly higher mental workload levels. A recent study showed that nurses showed excitability, irritability, unwillingness to rest, and signs of psychological distress in mobile hospitals (Chen et al., 2020). Thus, psychological interventions and assistance should be provided to nurses, whether online or face-to-face, in order to help decrease work-related stress.

Fatigue is also a critical factor involved in work-related errors and burnout (Yu et al., 2016). The prevalence of fatigue among healthcare workers varies between $21.6 \%$ and $91.9 \%$ (Cai et al., 2018). Our current study showed significant differences in fatigue among nurses belonging to the different subgroups. Multivariate analysis further demonstrated that high cognitive workload significantly increased the subjective assessment of fatigue, regardless of demographic variables such as age, gender, marital state and professional years. This result is in line with a previous study that showed excess cognitive workload resulted in fatigue, independent of inadequate sleep (Goel et al., 2014). Theoretically, high mental workloads can result in increased physical fatigue and mental distress in a clinical setting, which may negatively affect nurses' work engagement (Austin et al., 2020). On the contrary, our current study showed that nurses with higher UWES scores belonged to the high workload perception \& low selfevaluation group. Thus, there may be a potential positive correlation between nurses' mental workload and work engagement. Moreover, a positive and supportive working environment can often accommodate nurses' psychological needs and provide greater work engagement, which requires further investigation.

\section{Limitations}

There are several limitations with the current study. Firstly, a convenience sample was used, and therefore may possibly have limited the generalizability of the study population. Secondly, our study utilized selfreported data exclusively, and therefore may have potential social desirability bias. Thirdly, as a subjective scale, the NASA-TLX has been reported to have lower ecological validity than objective methods for assessing mental workload. In addition, assessment of workload during the working hours may have adverse influence by being perceived as an additional stressor. Thus, we cannot rule out the possible influence of various factors faced by the nurses when filling out the scales. Future studies may opt to assess nurses' workload by other means, such as workplace observations, supervisor ratings or objective performance indicators.

\section{Conclusions}


The current study examined the mental workload patterns and responses faced by nurses during the COVID-19 outbreak. A total of $62.5 \%$ of participants belonged to class 3 , that is, those who reported to be the most fatigued. High COVID-19 exposure risk was associated with higher mental workload in nurses. The complex scenario for the care of patients with infectious diseases, especially during an epidemic, raises the need for improved consideration of nurses' perceived workload, as well as their physical fatigue, work engagement and personal safety. Demographic factors may have variations based on the type of mental workload and may pose a challenge for health institutions in adapting to the changing needs of the environment, and require improved human resource management strategies that are not only patient-centered, but also safe and efficient for nurses.

\section{Abbreviations}

COVID-19: coronavirus disease 2019; LCA: latent class analysis; ANCOVA: analysis of covariance; NASATLX: National Aeronautics and Space Administration Task Load Index; FS-14: The Fatigue Scale-14; UWES: Utrecht Work Engagement Scale; AIC: Akaike information criterion; BIC: Bayesian information criterion; ABIC: sample-size adjustment BIC; LMR-LRT: the Lo-Mendell-Rubin likelihood ratio test; MCA: multiple correspondence analysis; PPE: personal protective equipment

\section{Declarations}

\section{Availability of data and materials}

The dataset and analyses are not currently publicly available; however, the materials could be available from the corresponding author on reasonable request.

\section{Ethics approval and consent to participate}

This study was approved by Shanghai University of Traditional Chinese Medicine Research Ethics Committee (Reg. no. 2020SHL-KY-025) and followed the national and local ethical standards. Informed consent was obtained from all study participants.

\section{Consent for publication}

Not applicable.

\section{Competing interests}

None declared.

\section{Funding}


No external funding for the research, authorship and/or publication of this paper.

\section{Authors' contributions}

WJ contributed to participate in the design, planning and development of the study. LHS were the main responsible for drafting the manuscript. WYM and WX contributed to data collection, GZH and LHS contributed to the analysis and interpretation of the data. GZH, WYM and WX who thoroughly read and amended the drafts. The final manuscript was read and approved by WJ and ZJ. The authors read and approved the final manuscript.

\section{Acknowledgements}

The authors would like to thank the relevant department leaders for their assistance to get in contact with the participating first-line nurses. We would also gratefully acknowledge the nurses who participated in this study.

\section{References}

1. Austin S, Fernet C, Trépanier S, Lavoie-Tremblay M. Fatigue in new registered nurses: A 12-month cross-lagged analysis of its association with work motivation, engagement, sickness absence and turnover intention. J Nurs Adm Manag. 2020;28(3):606-14. https://doi.org/10.1111/jonm.12962.

2. Cai S, Lin H, Hu X, Cai Y, Chen K, Cai W. High fatigue and its associations with health and work related factors among female medical personnel at 54 hospitals in Zhuhai, China. Psychology health medicine. 2018;23(3):304-16. https://doi.org/10.1080/13548506.2017.1361038.

3. Chalder T, Berelowitz G, Pawlikowska T, Watts L, Wessely S, Wright D, Wallace E. Development of a fatigue scale. J Psychosom Res. 1993;37(2):147-53. https://doi.org/10.1016/0022-3999(93)90081p.

4. Charles R, Nixon J. Measuring mental workload using physiological measures: A systematic review. Applied ergonomics. 2019;74:221-32. https://doi.org/10.1016/j.apergo.2018.08.028.

5. Chen I, Brown R, Bowers B, Chang W. Job Demand and Job Satisfaction in Latent Groups of Turnover Intention Among Licensed Nurses in Taiwan Nursing Homes. Res Nurs Health. 2015;38(5):342-56. https://doi.org/10.1002/nur.21667.

6. Chen Q, Liang M, Li Y, Guo J, Fei D, Wang L, He L, Sheng C, Cai Y, Li X, Wang J, Zhang Z. Mental health care for medical staff in China during the COVID-19 outbreak. The lancet Psychiatry. 2020;7(4):e15-6. https://doi.org/10.1016/S2215-0366(20)30078-X.

7. Collet C, Salvia E, Petit-Boulanger C. Measuring workload with electrodermal activity during common braking actions. Ergonomics. 2014;57(6):886-96. https://doi.org/10.1080/00140139.2014.899627. 
8. Donders N, Bos J, van der Velden K, van der Gulden J. Age differences in the associations between sick leave and aspects of health, psychosocial workload and family life: a cross-sectional study. BMJ open. 2012;2(4):e000960. https://doi.org/10.1136/bmjopen-2012-000960.

9. Fang L, Shi K, Zhang F. Research on reliability and validity of Utrecht Work Engagement Scalestudent. Chinese Journal of Clinical Psychology. 2008;16(6):618-20.

10. Felton E, Williams J, Vanderheiden G, Radwin R. Mental workload during brain-computer interface training. Ergonomics. 2012;55(5):526-37. https://doi.org/10.1080/00140139.2012.662526.

11. Galy E, Paxion J, Berthelon C. Measuring mental workload with the NASA-TLX needs to examine each dimension rather than relying on the global score: an example with driving. Ergonomics. 2018;61(4):517-27. https://doi.org/10.1080/00140139.2017.1369583.

12. Goel N, Abe T, Braun M, Dinges D. Cognitive workload and sleep restriction interact to influence sleep homeostatic responses. Sleep. 2014;37(11):1745-56. https://doi.org/10.5665/sleep.4164.

13. Happell B, Dwyer T, Reid-Searl K, Burke K, Caperchione C, Gaskin C. Nurses and stress: recognizing causes and seeking solutions. J Nurs Adm Manag. 2013;21(4):638-47. https://doi.org/10.1111/jonm.12037.

14. Hart S, Staveland L. (1988). Development of NASA-TLX (Task Load Index): Results of empirical and theoretical research. North-Holland. pp. 139-183.

15. Hart S. (2006). NASA-task load index (NASA-TLX); 20 years later. Los Angeles, CA: Sage publications. pp. 904-908.

16. Hoonakker P, Carayon P, Gurses A, Brown R, McGuire K, Khunlertkit A, Walker J. MEASURING WORKLOAD OF ICU NURSES WITH A QUESTIONNAIRE SURVEY: THE NASA TASK LOAD INDEX (TLX). IIE transactions on healthcare systems engineering. 2011;1(2):131-43. https://doi.org/10.1080/19488300.2011.609524.

17. Houghton C, Meskell P, Delaney H, Smalle M, Glenton C, Booth A, Chan X, Devane D, Biesty L. Barriers and facilitators to healthcare workers' adherence with infection prevention and control (IPC) guidelines for respiratory infectious diseases: a rapid qualitative evidence synthesis. Cochrane Database Syst Rev. 2020;4(4):CD013582. https://doi.org/10.1002/14651858.CD013582.

18. Huang L, Lin G, Tang L, Yu L, Zhou Z. Special attention to nurses' protection during the COVID-19 epidemic. Critical care. 2020;24(1):120. https://doi.org/10.1186/s13054-020-2841-7.

19. Jaquess K, Lo L, Oh H, Lu C, Ginsberg A, Tan Y, Lohse K, Miller M, Hatfield B, Gentili R. Changes in Mental Workload and Motor Performance Throughout Multiple Practice Sessions Under Various Levels of Task Difficulty. Neuroscience. 2018;393:305-18. https://doi.org/10.1016/j.neuroscience.2018.09.019.

20. Legenza L, Nickman N, Drews F, Rim M, Tigh J, Kelly M, Tyler L. Assessment of perceived workload in academic health center community pharmacies before and after implementation of a central call center. American journal of health-system pharmacy. 2019;76(21):1794-805. https://doi.org/10.1093/ajhp/zxz200. 
21. Liang L. Study on the Status of Nurses' Mental workload and Its Relationship with Psychological Capital and Coping Style Based on LPA. M.D.: PLA Naval Medical University; 2019.

22. Schaufeli W, Salanova M, González-Romá V, Bakker A. The measurement of engagement and burnout: A two sample confirmatory factor analytic approach. Journal of Happiness studies. 2002;3(1):71-92.

23. Tubbs-Cooley H, Mara C, Carle A, Gurses A. The NASA Task Load Index as a measure of overall workload among neonatal, paediatric and adult intensive care nurses. Intensive critical care nursing. 2018;46:64-9. https://doi.org/10.1016/j.iccn.2018.01.004.

24. Tubbs-Cooley H, Mara C, Carle A, Mark B, Pickler R. Association of Nurse Workload with Missed Nursing Care in the Neonatal Intensive Care Unit. JAMA pediatrics. 2019;173(1):44-51. https://doi.org/10.1001/jamapediatrics.2018.3619.

25. Xu C, Zheng Z, Zhang Z. The individual characters on nurse's occupational stress and fatigue. Chinese Journal of Nursing. 2006;41(6):530.

26. Young G, Zavelina L, Hooper V. Assessment of workload using NASA Task Load Index in perianesthesia nursing. Journal of perianesthesia nursing. 2008;23(2):102-10. https://doi.org/10.1016/j.jopan.2008.01.008.

27. Yu H, Jiang A, Shen J. Prevalence and predictors of compassion fatigue, burnout and compassion satisfaction among oncology nurses: A cross-sectional survey. International journal of nursing studies. 2016;57:28-38. https://doi.org/10.1016/j.jpurstu.2016.01.012.

28. Zhou F, Yu T, Du R, Fan G, Liu Y, Liu Z, Xiang J, Wang Y, Song B, Gu X, Guan L, Wei Y, Li H, Wu X, Xu J, Tu S, Zhang Y, Chen H, Cao B. Clinical course and risk factors for mortality of adult inpatients with COVID-19 in Wuhan, China: a retrospective cohort study. Lancet. 2020;395(10229):1054-62. https://doi.org/10.1016/S0140-6736(20)30566-3.

\section{Figures}




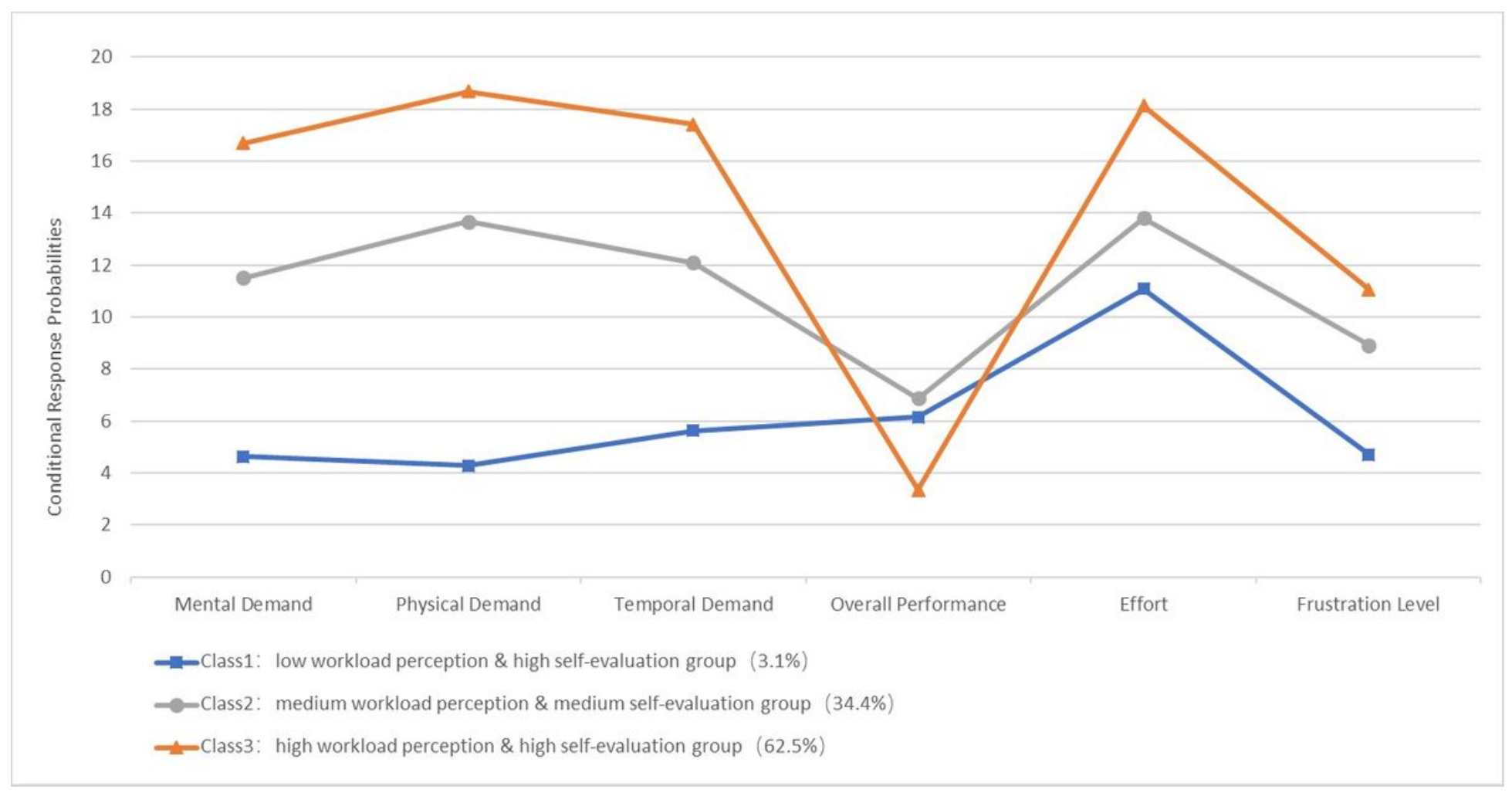

\section{Figure 1}

Three subtypes of nurses' mental workload based on the LCA results 


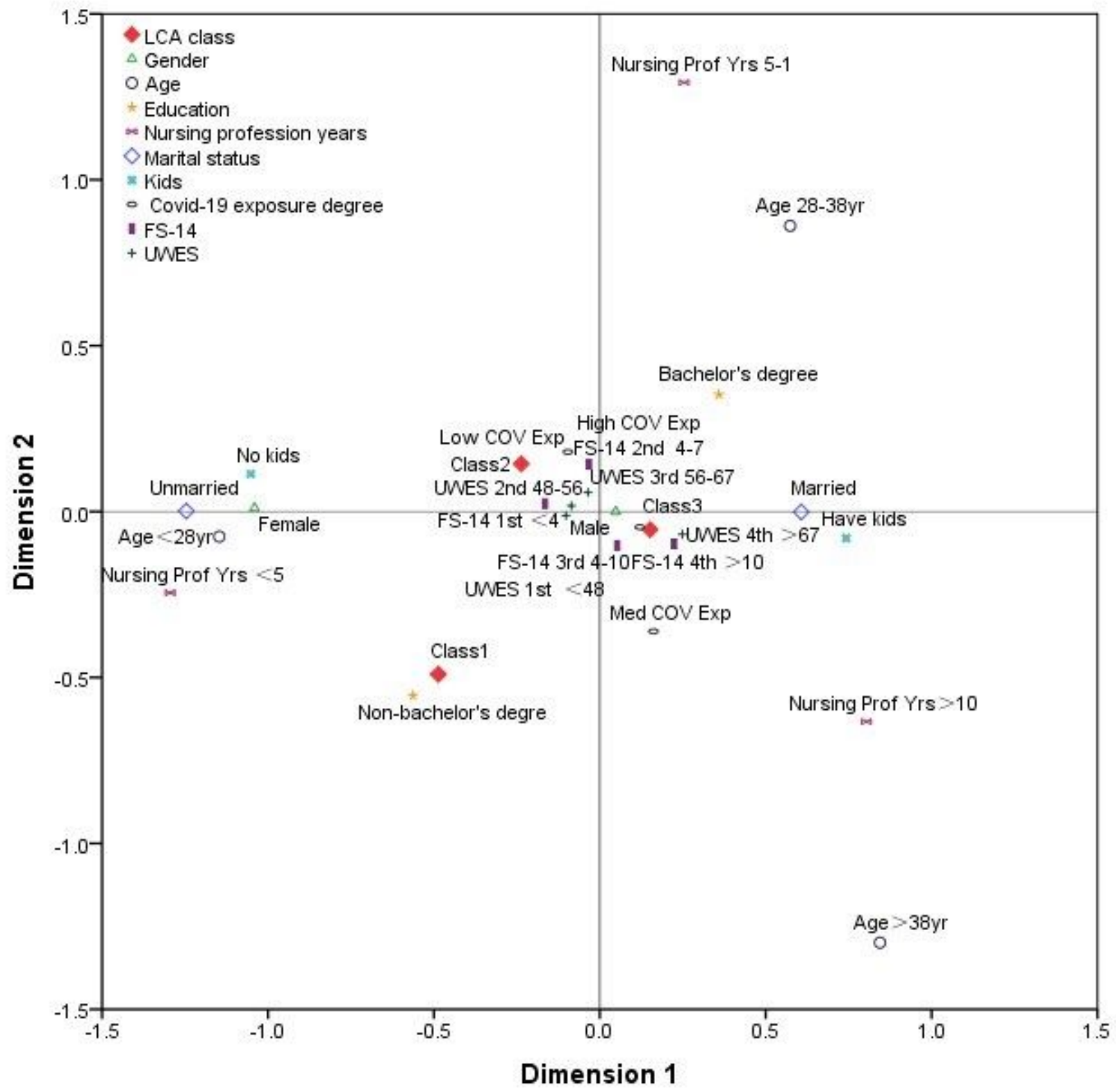

Figure 2

Multiple correspondence analysis plot The categorical variables entered into the multiple correspondence analysis (MCA) model (based upon optimal scaling) to evaluate the potential association with mental workload. Each variable was visualized with a specific icon and the brief description was labeled near the icon. Interpretation of this graphic output was mainly based on the distance between category-icons.

\section{Supplementary Files}

This is a list of supplementary files associated with this preprint. Click to download.

- STROBEchecklist.docx 